\title{
Addition of some bird species to checklist of Nandankanan wildlife sanctuary, Odisha
}

\section{Introduction}

Conservation of taxa in a given landscape starts with a sound knowledge on the species available or a checklist of the same. In the present days the growing interest on bird watching made the birds among the most studied taxa. Present communication reports addition of some bird species to the existing checklist of birds of Nandankanan Wildlife Sanctuary (NWS). NWS is located between $20^{\circ} 23^{\prime} 08^{\prime \prime}$ to $20^{\circ} 24^{\prime} 10^{\prime \prime} \mathrm{N}$ and $85^{\circ} 48^{\prime} 09^{\prime \prime}$ to $85^{\circ} 48^{\prime} 13^{\prime \prime} \mathrm{E}$, spreading over an area of 4.37 sq.km in the state of Odisha, Eastern India. The sanctuary comprises of Nandankanan Zoological Park, State Botanical Garden, Kanjia Lake: an wetland of national importance and forest lands with other small to medium wetlands. It has moist mixed deciduous vegetation and moderately hot humid climate with average temperature of $30^{\circ} \mathrm{C}$ with annual average rainfall of $1350 \mathrm{~mm}$.

\section{Methodology}

Field surveys were carried out ad libitum by the authors inside the sanctuary area between February 2014 and January 2019. Bird species were identified on site and confirmed following standard identification. $^{1-3}$

\section{Result and discussion}

Earlier studies have documented 124 species of birds ${ }^{4-7}$ at Nandankanan Wildlife Sanctuary (Figure 1) in the state Odisha, eastern India. The
Volume 4 Issue 3 - 2019

RK Mohapatra,' S Purohit, ${ }^{2}$ SS Ray, ${ }^{3}$ AA Sahoo, ${ }^{3}$ MK Panda,' BP Panda, ${ }^{4}$ S Nanda, ${ }^{5}$ M Sahoo, ${ }^{5}$ BK Padhee, ${ }^{5}$ M Parmar, ${ }^{5} \cup$ Biswal, ${ }^{4}$ SP Parida, ${ }^{5}$ JK Das, 'S Paul'

'Nandankanan Biological Park, India ${ }^{2}$ DBCNR, Central University of Orissa, India ${ }^{3}$ College of Forestry, OUAT, Odisha, India ${ }^{4}$ ITER, SOA Deemed University, Odisha, India ${ }^{5}$ Bhubaneswar, Odisha, India ${ }^{6}$ Centurion University of Technology \& Management, India

Correspondence: RK Mohapatra, Nandankanan Biological Park, Bhubaneswar-754005, Odisha, India, Email rajesh.wildlife@gmail.com

Received: February II, 2019 | Published: June 04, 2019

present communication reports additional 46 species of the birds sighted and/or photo-documented during the study period (Table 1), (Figure 2). Detailed study is needed to find out any additional species available, their seasonal migration, feeding and breeding biology.

Table I List of additional birds sighted in Nandankanan Wildlife Sanctuary

\begin{tabular}{|c|c|c|c|c|c|}
\hline $\begin{array}{l}\text { Sl. } \\
\text { No }\end{array}$ & Order & Family & Common name & $\begin{array}{l}\text { Scientific } \\
\text { name }\end{array}$ & Remarks \\
\hline $\mathrm{I}$. & Accipitriformes & Accipitridae & $\begin{array}{l}\text { Oriental honey } \\
\text { buzzard }\end{array}$ & $\begin{array}{l}\text { Pernis } \\
\text { ptilorhynchus }\end{array}$ & Sighted on 07.01 .2019 while it hovering above Kanjia lake \\
\hline 2. & Accipitriformes & Accipitridae & Black-winged kite & Elanus caeruleus & $\begin{array}{l}\text { Seen during bird census } 2019 \text { near Kanjia lake on } \\
03.01 .2019\end{array}$ \\
\hline 3. & Accipitriformes & Accipitridae & Besra & Accipiter virgatus & $\begin{array}{l}\text { Few sightings while it hovering above Badabanka pata, } \\
\text { Nandankanan and Hameratangi area, Nandankanan }\end{array}$ \\
\hline 4. & Accipitriformes & Accipitridae & Crested goshawk & Accipiter trivirgatus & $\begin{array}{l}\text { Few sightings while it hovering above Badabanka pata, } \\
\text { Nandankanan and Hameratangi area, Nandankanan }\end{array}$ \\
\hline 5. & Accipitriformes & Accipitridae & $\begin{array}{l}\text { Eurasian Marsh } \\
\text { Harrier }\end{array}$ & Circus aeruginosus & $\begin{array}{l}\text { One individual sighted hovering near Kanjia lake on } \\
27.01 .2017\end{array}$ \\
\hline 6. & Charadriiformes & Scolopacidae & Common red shank & Tringa totanus & Sighted near Kanjia lake area on 10.01.2018 \\
\hline 7. & Charadriiformes & Scolopacidae & Common sandpiper & Actitis hypoleucos & Sighted near Kanjia lake area on I0.01.2018 \\
\hline 8. & Charadriiformes & Scolopacidae & Marsh sandpiper & Tringa stagnatilis & Sighted near Kanjia lake area on 10.01.2018 \\
\hline 9. & Charadriiformes & Charadriidae & $\begin{array}{l}\text { Grey headed } \\
\text { lapwing }\end{array}$ & Vanellus cinereus & Sighted near Kanjia lake area on 10.01.2018 \\
\hline 10. & Charadriiformes & Charadriidae & River lapwing & Vanellus duvaucelii & Sighted near Kanjia lake area on 10.01 .2018 \\
\hline $\mathrm{II}$ & Charadriiformes & Laridae & River tern & Sterna aurantia & Seen during bird census 2019 on 03.01 .2019 \\
\hline 12. & Coraciiformes & Alcedinidae & $\begin{array}{l}\text { Black capped } \\
\text { Kingfisher }\end{array}$ & Halcyon pileata & $\begin{array}{l}\text { One individual observed on } 23.03 .2013 \text { near holiday } \\
\text { cottage of State Botanical Garden }\end{array}$ \\
\hline 13. & Columbiformes & Columbidae & $\begin{array}{l}\text { Yellow-footed green } \\
\text { pigeon }\end{array}$ & $\begin{array}{l}\text { Treron } \\
\text { phoenicoptera }\end{array}$ & Six individuals were observed on 17.02 .2018 \\
\hline 14. & Columbiformes & Columbidae & $\begin{array}{l}\text { Orange-breasted } \\
\text { green pigeon }\end{array}$ & Treron bicinctus & Few sightings at State Botanical Garden \\
\hline
\end{tabular}


Table Continued

\begin{tabular}{|c|c|c|c|c|c|}
\hline $\begin{array}{l}\text { SI. } \\
\text { No }\end{array}$ & Order & Family & Common name & $\begin{array}{l}\text { Scientific } \\
\text { name }\end{array}$ & Remarks \\
\hline 15. & Cuculiformes & Cuculidae & $\begin{array}{l}\text { Chestnut winged } \\
\text { cuckoo }\end{array}$ & $\begin{array}{l}\text { Clamator } \\
\text { coromandus }\end{array}$ & $\begin{array}{l}\text { Two sightings in State Botanical Garden area one in June, } \\
2014 \text { and other January, } 2015\end{array}$ \\
\hline 16. & Cuculiformes & Cuculidae & Grey-bellied cuckoo & $\begin{array}{l}\text { Cacomantis } \\
\text { passerinus }\end{array}$ & $\begin{array}{l}\text { Observed near Children park, Nandankanan during bird } \\
\text { census } 2017\end{array}$ \\
\hline 17. & Falconiformes & Falconidae & Common Kestrel & Falco tinnunculus & Sighted near Hamera tangi area on 10.01 .2018 \\
\hline 18. & Gruiformes & Rallidae & Baillon's Crake & Zapornia pusilla & One individual sighted in the swampy bush of Kanja lake \\
\hline 19. & Passeriformes & Hirundinidae & Wire-tailed swallow & Hirundo smithii & Frequently observed during the study period \\
\hline 20. & Passeriformes & Hirundinidae & $\begin{array}{l}\text { Red-rumped } \\
\text { swallow }\end{array}$ & Cecropis daurica & Frequently observed during the study period \\
\hline 21. & Passeriformes & Cisticolidae & Plain prinia & Prinia inornata & Frequently observed during the study period \\
\hline 22. & Passeriformes & Acrocephalidae & $\begin{array}{l}\text { Clamorous reed } \\
\text { warbler }\end{array}$ & $\begin{array}{l}\text { Acrocephalus } \\
\text { stentoreus }\end{array}$ & One individual sighted near bush of kanjia lake 19.11.2016 \\
\hline 23. & Passeriformes & Sturnidae & $\begin{array}{l}\text { Chestnut tailed } \\
\text { starling }\end{array}$ & Sturnia malabarica & Frequently observed during the study period \\
\hline 24. & Passeriformes & Sturnidae & Rosy starling & Pastor roseus & $\begin{array}{l}\text { A flock observed near Mughal garden of State Botanical } \\
\text { Garden during summer season } 2017\end{array}$ \\
\hline 25. & Passeriformes & Muscicapidae & $\begin{array}{l}\text { White rumped } \\
\text { shama }\end{array}$ & $\begin{array}{l}\text { Copsychus } \\
\text { malabaricus }\end{array}$ & Frequently observed during the study period \\
\hline 26. & Passeriformes & Muscicapidae & $\begin{array}{l}\text { Asian brown } \\
\text { flycatcher }\end{array}$ & $\begin{array}{l}\text { Muscicapa } \\
\text { latirostris }\end{array}$ & Frequently observed during the study period \\
\hline 27. & Passeriformes & Muscicapidae & $\begin{array}{l}\text { Asian verditer } \\
\text { flycatcher }\end{array}$ & $\begin{array}{l}\text { Eumyias } \\
\text { thalassinus }\end{array}$ & Frequently observed during the study period \\
\hline 28. & Passeriformes & Muscicapidae & $\begin{array}{l}\text { Red breasted } \\
\text { flycatcher }\end{array}$ & Ficedula parva & $\begin{array}{l}\text { Frequently observed behind the butterfly garden after } \\
\text { 19.11.2016 }\end{array}$ \\
\hline 29. & Passeriformes & Muscicapidae & $\begin{array}{l}\text { Blue throated blue } \\
\text { flycatcher }\end{array}$ & $\begin{array}{l}\text { Cyornis } \\
\text { rubeculoides }\end{array}$ & $\begin{array}{l}\text { One individual observed near Butterfly garden of State } \\
\text { Botanical Garden on 19.11.2016 }\end{array}$ \\
\hline 30. & Passeriformes & Motacillidae & Grey wagtail & Motacilla cinerea & Seen during winter season 2017 \\
\hline 31. & Passeriformes & Motacilidae & Citrine wagtail & Motacilla citreola & Sighted near Kanjia lake on 10.01.2018 \\
\hline 32. & Passeriformes & Motacilidae & Yellow wagtail & Motacilla flava & Sighted near Kanjia lake area on 07.01.2019 \\
\hline 33. & Passeriformes & Oriolidae & Black naped oriole & Oriolus chinensis & $\begin{array}{l}\text { It had been frequently observed on large trees during } \\
\text { winter months }\end{array}$ \\
\hline 34. & Passeriformes & Muscicapidae & Blue rock thrush & $\begin{array}{l}\text { Monticola } \\
\text { solitarius }\end{array}$ & Sighted in State Botanical Garden \\
\hline 35. & Passeriformes & Muscicapidae & Pied bushchat & Saxicola caprata & Frequently observed during the study period \\
\hline 36. & Passeriformes & Motacillidae & Olive backed pipit & Anthus hodgsoni & Seen in State Botanical Garden on 22.10.2016 \\
\hline 37. & Passeriformes & Laniidae & Long tailed shrike & Lanius schach & $\begin{array}{l}\text { Sighted near Hamera tangi area, Nandankanan on } \\
07.01 .2019\end{array}$ \\
\hline 38. & Passeriformes & Acrocephalidae & Booted warbler & Iduna caligata & $\begin{array}{l}\text { Sighted near Hamera tangi area, Nandankanan on } \\
03.01 .2019\end{array}$ \\
\hline 39. & Passeriformes & Hirundinidae & House martin & Delichon urbicum & $\begin{array}{l}\text { Sighted near Hamera tangi area, Nandankanan on } \\
03.01 .2019\end{array}$ \\
\hline 40. & Passeriformes & Estrildidae & Spotted munia & $\begin{array}{l}\text { Lonchura } \\
\text { punctulata }\end{array}$ & $\begin{array}{l}\text { Frequently observed to be nesting in the Cycas plants at } \\
\text { Forest rest house, Nandankanan }\end{array}$ \\
\hline 41. & Passeriformes & Campephagidae & Small minivet & $\begin{array}{l}\text { Pericrocotus } \\
\text { cinnamomeus }\end{array}$ & $\begin{array}{l}\text { One male observed on } 16.02 .2014 \text { near Hamera tangi } \\
\text { area, Nandankanan }\end{array}$ \\
\hline 42. & Passeriformes & Cisticolidae & Ashy prinia & Prinia socialis & Frequently observed during the study period \\
\hline 43. & Pelecaniformes & Ciconiidae & $\begin{array}{l}\text { Lesser adjutant } \\
\text { stork }\end{array}$ & $\begin{array}{l}\text { Leptoptilos } \\
\text { javanicus }\end{array}$ & $\begin{array}{l}\text { Sighted near Hamera tangi area, Nandankanan on } \\
\text { I0.01.20I8 }\end{array}$ \\
\hline 44. & Piciformes & Picidae & $\begin{array}{l}\text { Fulvous-breasted } \\
\text { woodpecker }\end{array}$ & $\begin{array}{l}\text { Dendrocopos } \\
\text { macei }\end{array}$ & $\begin{array}{l}\text { Sighted in Hamera tangi area, Nandankanan and State } \\
\text { Botanical Garden }\end{array}$ \\
\hline 45. & Phoenicopteriformes & Podicipedidae & Little grebe & $\begin{array}{l}\text { Tachybaptus } \\
\text { ruficollis }\end{array}$ & Two individuals seen in Kanjia lake on 26.12.20I5 \\
\hline 46. & Strigiformes & Strigidae & Brown hawk owl & Ninox scutulata & $\begin{array}{l}\text { One individual observed on } 05.10 .13 \text { in State Botanical } \\
\text { Garden }\end{array}$ \\
\hline
\end{tabular}




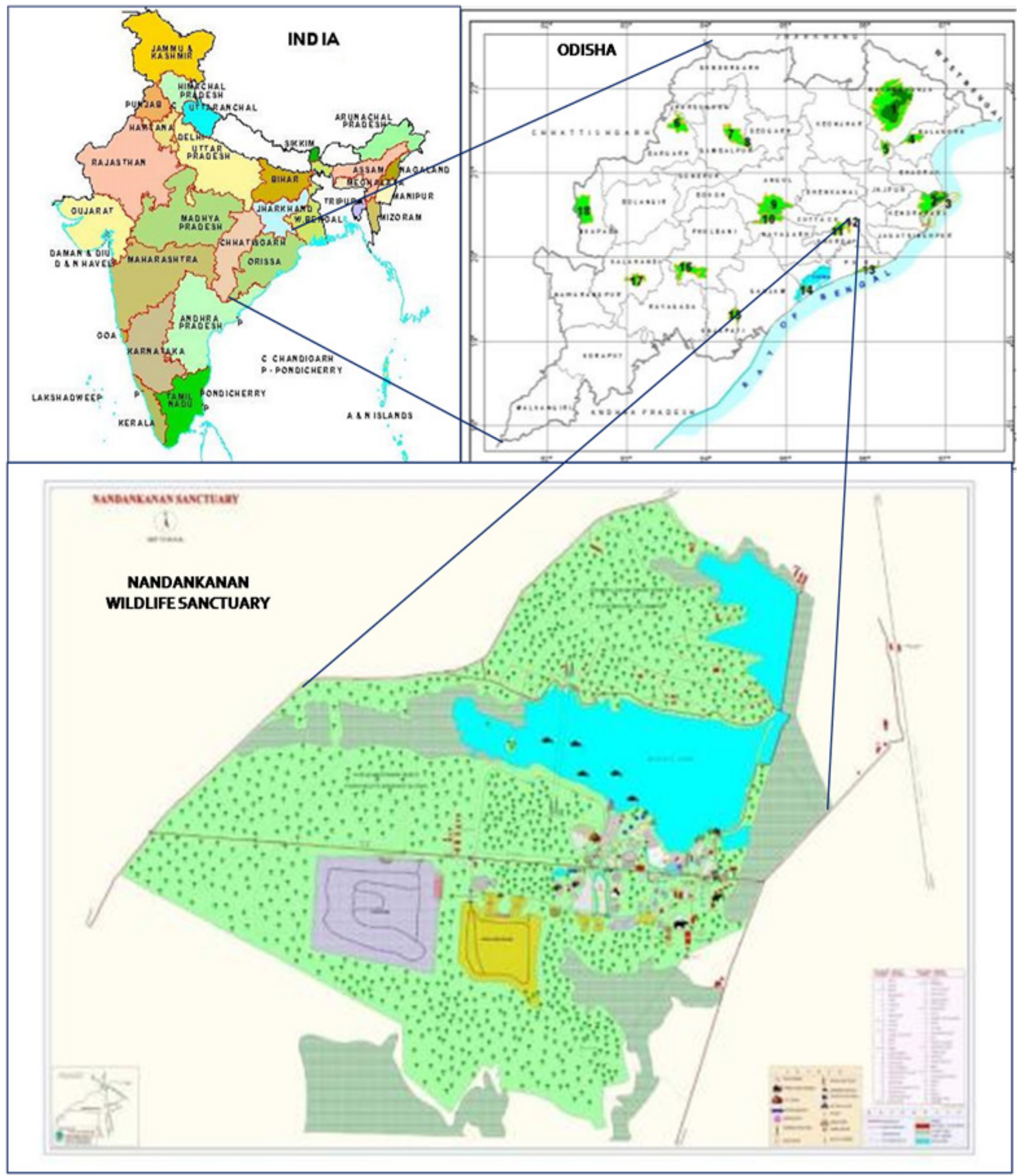

Figure I Nandankanan Wildlife Sanctuary, Odisha, India.

Copy right of images:The photographs are taken by the authors (RKM:2,8, I I, I3; SP: 18, I9; SSR:I, 3-6, I0, I2,32;AAS: 14- I7, 20-30; MKP:7,33; BPP:3।; SN:9; BKP:3; UB:34-36) 


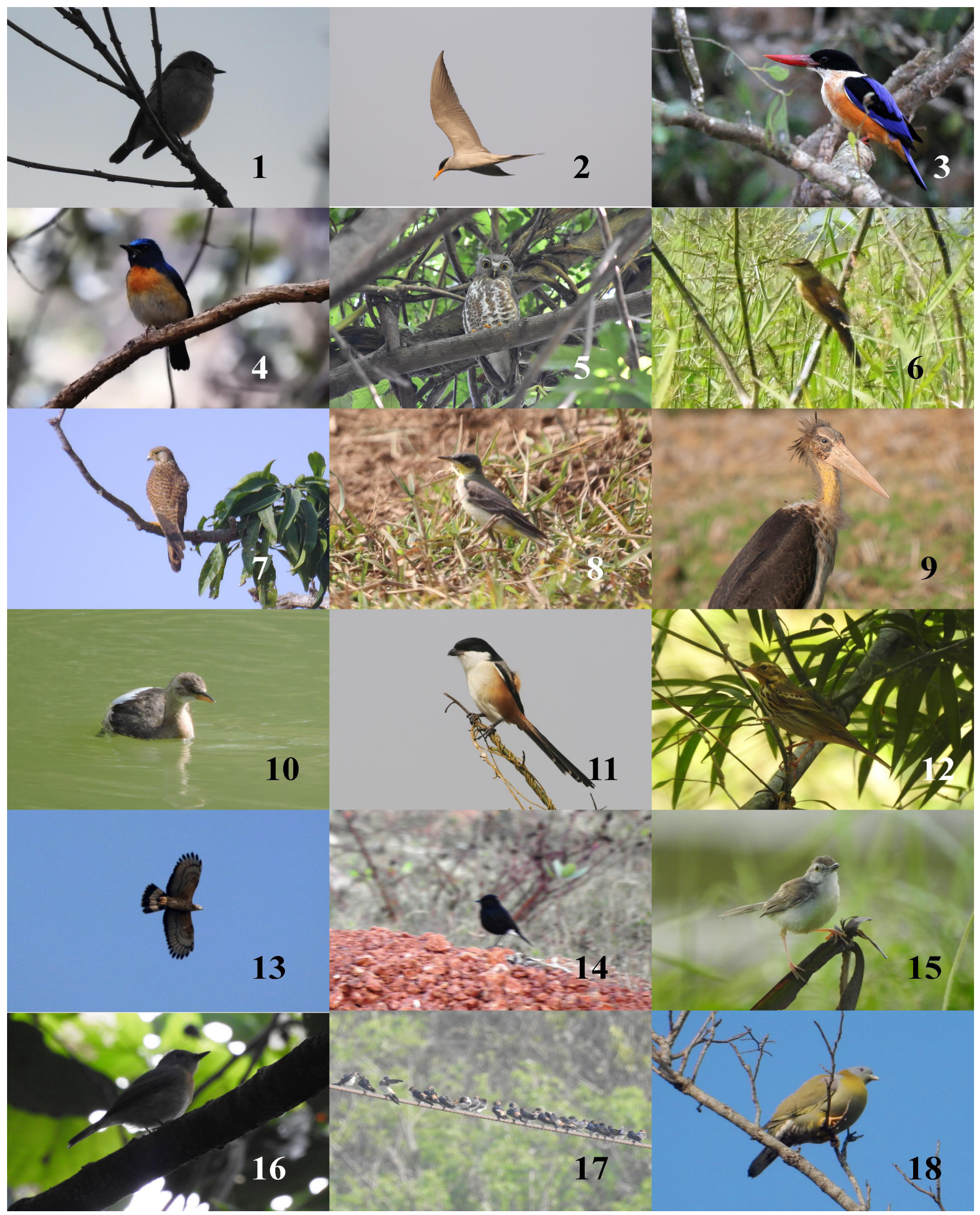

Citation: Mohapatra RK, Purohit S, Ray SS, et al.Addition of some bird species to checklist of Nandankanan wildlife sanctuary, Odisha. Int J Avian \& Wildlife Biol. 2019;4(3):80-85. DOI: I0.15406/ijawb.2019.04.00156 


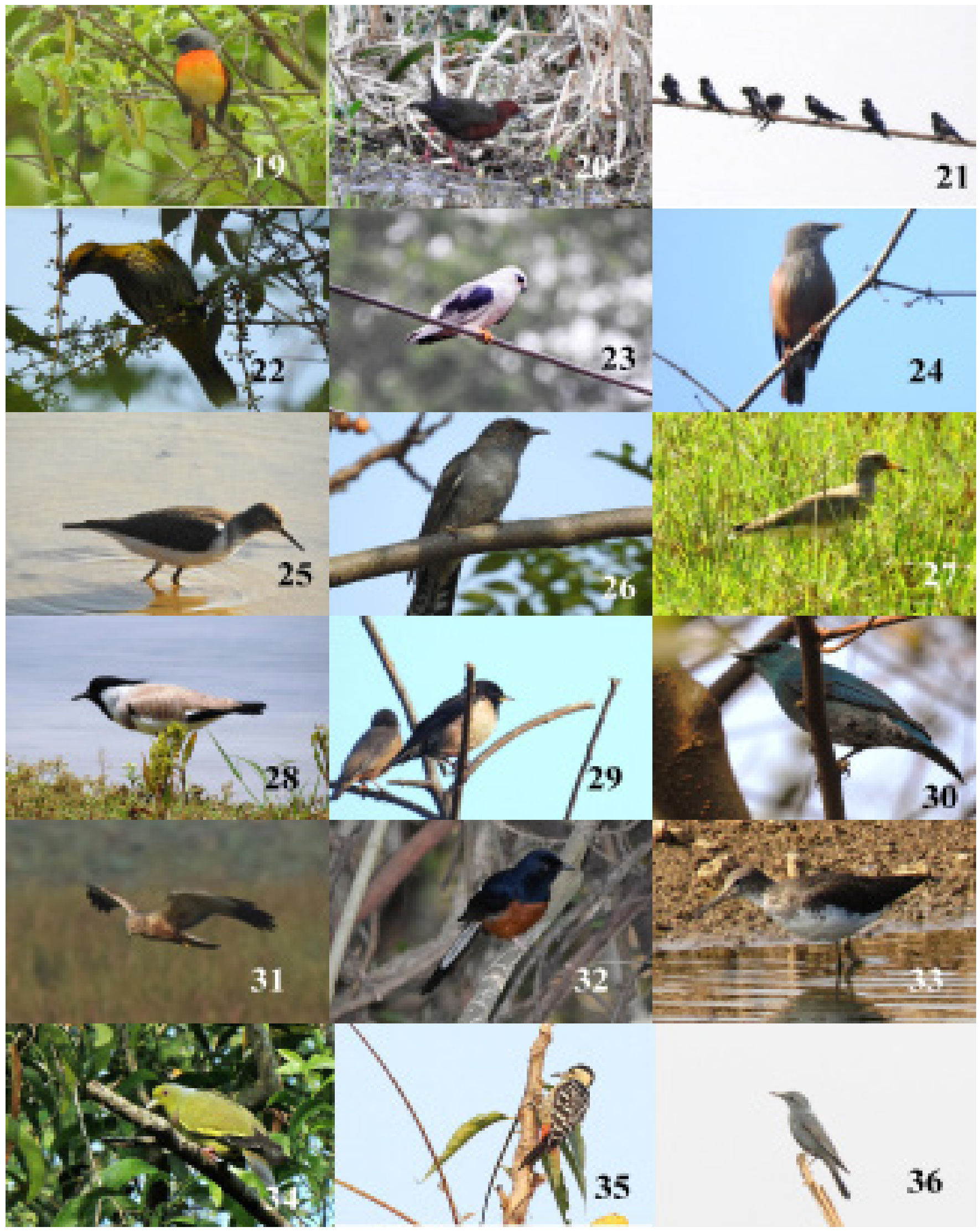

Citation: Mohapatra RK, Purohit S, Ray SS, et al.Addition of some bird species to checklist of Nandankanan wildlife sanctuary, Odisha. Int J Avian \& Wildlife Biol. 2019;4(3):80-85. DOI: I0.15406/ijawb.2019.04.00156 
Figure 2 Photographs of some species sighted (I-Asian brown flycatcher, 2-River tern, 3-Black capped kingfisher, 4-Blue throated blue flycatcher, 5-Brown hawk owl, 6-Clamorous reed warbler, 7-Common kristel, 8-Grey wagtail, 9-Lesser adjutant, I0-Lettle grebe, II-Long tailed shrike, I2-Olive backed pipit, 13-Oriental honey buzzard, I4-Pied bushchat, I5-Plain prinia, 16-Red breasted flycatcher, I7- Red-rumped swallow, I8- Yellow-footed green pigeon, I9-Small minivet, 20-Baillon's crake, 21-Wire tailed swallow, 22-Black naped oriole, 23-Black winged kite, 24-Chestnut tailed starling, 25-Common sandpiper, 26-Grey bellied cuckoo, 27-Grey headed lapwing, 28-River lapwing, 29-Rosy starling, 30-Verdicter flycatcher, 31-Western marsh harrier, 32-White rumped shama, 33Wood sandpiper, 34- Orange-breasted green pigeon, 35-Fulvous-breasted woodpecker, 36- Blue rock thrush).

Copy right of images:The photographs are taken by the authors (RKM: 2,8, I I, I3; SP:I8, I 9; SSR:I, 3-6, I0, I2,32;AAS: I4-I 7,20-30; MKP:7,33; BPP:3 I; SN:9; BKP:3; UB:34-36)

\section{Acknowledgments}

None.

\section{Conflicts of interest}

Author declares that there are no conflicts of interest.

\section{Reference}

1. Grimmett R, Inskipp C, Inskipp T. Helm field guides Birds of the Indian subcontinent. $2^{\text {nd }}$ edition. Delhi: Oxford University Press; 2011. pp. $1-528$.

2. Kamal S, Indresh GN, Mishra AK. Vertebrate faunal diversity of Kanjia Lake, Nandankanan. e-planet. 2009;7(2):29-33.

3. Kazmierczak K. A field guide to the birds of the Indian Subcontinent London: Bloomsbury publishing; 2015. p. 352.
4. Mohapatra RK, Panda S, Purohit KL. Wintering avian population of Nandankanan wildlife sanctuary, Odisha. Tigerpaper. 2013;40(2):31-34.

5. Mohapatra RK. Sighting of Indian grey hornbill and Rufous woodpecker at Nandankanan Wildlife Sanctuary, Odisha, India. Int J Avian \& Wildlife Biol. 2018;3(4):307-308.

6. Panda S, Mishra S, Mishra AK, et al. Nandankanan Faunal Diversity. Odisha: Nandankanan Biological Park, Forest and Environment Department, Government of Odisha; 2010. p. 152.

7. Rasmussen PC, Anderton JC. Birds of South Asia. The Ripley Guide. $2^{\text {nd }}$ Edition. Washington: National Museum of Natural History-Smithsonian Institution, Michigan State University and Lynx Edicions; 2012. p. 1-683. 